\title{
PORTACAVAL ANASTOMOSIS-INDUCED HYPERAMMONEMIA DOES NOT LEAD TO OXIDATIVE STRESS
}

\author{
Xiaoling Yang1, Cristina R. Bosoi ${ }^{1}$, Wenlei Jiang${ }^{1}$, Mélanie Tremblay ${ }^{1}$ and \\ Christopher F. Rose ${ }^{1}$
}

(1)Neuroscience Research Unit, Université de Montréal (CRCHUM), 1058 St-Denis Street, Montreal, Quebec, H2X 3J4, Canada Email: christopher.rose@umontreal.ca

\section{ABSTRACT}

Ammonia is neurotoxic and believed to play a major role in the pathogenesis of hepatic encephalopathy (HE). It has been demonstrated, in vitro and in vivo, that acute and high ammonia treatment induces oxidative stress. Reactive oxygen species (ROS) are highly reactive and can lead to oxidization of proteins resulting in protein damage. The present study was aimed to assess oxidative status of proteins in plasma and brain (frontal cortex) of rats with 4week portacaval anastomosis (PCA). Markers of oxidative stress, 4-hydroxy-2-nonenal (HNE) and carbonylation were evaluated by immunoblotting in plasma and frontal cortex. Western blot analysis did not demonstrate a significant difference in either HNE-linked or carbonyl derivatives on proteins between PCA and sham-operated control rats in both plasma and frontal cortex. The present study suggests PCA-induced hyperammonemia does not lead to systemic or central oxidative stress.

\section{Keywords}

Hepatic encephalopathy PCA Oxidative stress Hyperammonemia

\section{INTRODUCTION}

The brain relies on a healthy liver to detoxify circulating toxins which are capable of entering the brain and leading to functional impairment and hepatic encephalopathy (HE) (Butterworth 2003). HE is characterized with a spectrum of symptoms ranging from personality and mood changes, night-time sleep disturbances, alterations in concentration and attention, severe cognitive effects, coma and death (Hazell and Butterworth 1999). The pathogenesis of HE is complex, involving a number of possible toxins and neurotransmitter dysfunctions associated with a background of astrocytic pathology (Blendis 2006). Liver failure or disease reduces the capacity of the liver to remove neurotoxins such as ammonia, a key factor involved in the pathogenesis of HE (Bosoi and Rose 2009; Butterworth 2003).

The brain is susceptible to oxidative stress due to its high content of unsaturated fatty acids, high oxygen consumption, high levels of free radicals and relatively low levels of antioxidant defence systems (Choi and Yu 1995; Poon et al. 2004). Brain tissue or cells are constantly exposed to diverse reactions generating reactive oxygen species (ROS) that oxidize proteins, lipids and nucleic acids. Carbonyl derivatives, a product of protein oxidation, reflect cell modifications induced by multiple forms of ROS. Oxidative modification of proteins can also give rise to intra- or inter- protein cross-linked derivatives, demonstrated by reactions with the lipid peroxidation product 4-hydroxy-2noneal (HNE). As a result, cleavage of the polypeptide chain, modification of amino acid side chains, and conversion 
Yang, X. et al., 2010. Portacaval anastomosis-induced hyperammonemia does not lead to oxidative stress. Metabolic Brain Disease, 25(1), p.11-15.

of protein to derivatives that are highly sensitive to proteolytic degradation, lead to protein inactivation (Stadtman and Levine 2003).

Hyperammonemia is a metabolic disturbance characterized by an increase in blood ammonia which consequently results in an increase in brain ammonia (Felipo and Butterworth 2002). Recent studies have demonstrated acute ammonia neurotoxicity induces oxidative stress (Norenberg et al. 2004). Studies using cultured astrocytes have demonstrated an acute treatment of $5 \mathrm{mM}$ ammonia induces oxidative stress (Norenberg et al. 2007; Warskulat et al. 2002). In animal models of acute liver failure, ammonia-induced oxidative stress has also been observed (Reddy et al. 2004; Sathyasaikumar et al. 2007). Acute intoxication with high concentrations of ammonia $12 \mathrm{mmol} / \mathrm{kg}$ (intraperitoneally) or $60 \mathrm{mM}$ (intrastriatally) in rats has been shown to generate free radicals (Hilgier et al. 2003; Kosenko et al. 2003). Overall, studies demonstrating ammonia-induced oxidative stress have been involved in either acute liver failure or acute ammonia intoxication models. However the role of ammonia and oxidative stress in HE associated with chronic liver failure remains undefined. Using a 4-week portal-systemic shunting rat model, our aim was to investigate the role of oxidative stress in this chronic hyperammonemia, Type B minimal HE model (Butterworth et al. 2009). To determine the effect of oxidative stress in the systemic circulation and central nervous system, ROS-induced protein modifications, HNE-linked proteins and carbonyl detection, were assessed in arterial plasma and frontal cortex of rats with portacaval anastomosis (PCA).

\section{MATERIALS AND METHODS}

\section{Animal model}

Five male Sprague-Dawley rats (250-275 g) underwent end-to-side PCA, according to Lee and Fisher (1961). Briefly, laparotomy was performed and inferior vena cava and portal vein were isolated. The portal vein was ligated and cut. The inferior vena cava was clamped and an elliptical piece 1.5 times the diameter of the portal vein was removed. The end-to-side PCA was performed under a surgical microscope in less than 15 min. Five control rats of the same sex and age underwent a sham operation where the inferior vena cava and portal vein were isolated and clamped for 15 min, without performing the end-to-side PCA. All rats were sacrificed 4 weeks after surgery by decapitation.

\section{Cerebrospinal fluid (CSF) sampling}

CSF was collected by inserting a catheter into the cisterna magna. The rat was anesthetised with isoflurane and the skull was immobilized in a stereotaxic apparatus. An incision was made on the back of the head and the occipital bone was exposed. A dental burr was used to drill a hole on the saggital midline rostral to the interparietal-occipital bone suture. A PE-10 tubing catheter was inserted into the cisterna magna through the dura mater. Silastic tubing was attached to the catheter and to a $22 \mathrm{G}^{1 / 2}$ needle connected to a syringe. CSF was collected and immediately frozen in deeply cooled isopentane. After this procedure, arterial plasma and brain samples were collected, immediately frozen and stored at $-80^{\circ} \mathrm{C}$.

Experiments were conducted following the Guidelines of Canadian Council on Animal Care and were approved by the Animal Protection Committee of CHUM Research Center.

\section{Measurement of ammonia concentration}

Ammonia concentrations were measured in arterial plasma and CSF using a commercial kit (Sigma, St. Louis, MO, USA). Ammonia concentrations were assessed based on the reaction with $\alpha$-ketoglutarate and reduced nicotinamide adenine dinucleotide phosphate (NADPH) in presence of L-glutamate dehydrogenase. Oxidation rate of NADPH was recorded by the decrease in absorbance at $340 \mathrm{~nm}$. Ammonia concentration was calculated according to the manufacturer's protocol.

Data are expressed as mean \pm standard error of the mean (SEM). Significance of difference was tested with the Student t-test using GraphPad Prism 4 (La Jolla, CA, USA). Probability values of $\mathrm{p}<0.05$ were considered to be statistically significant.

The final publication is available at http://dx.doi.org/10.1007/s11011-010-9174-1 
Yang, X. et al., 2010. Portacaval anastomosis-induced hyperammonemia does not lead to oxidative stress. Metabolic Brain Disease, 25(1), p.11-15.

\section{Tissue preparation}

Frontal cortex (PCA or sham [n = 5]) was homogenized in lysis buffer (50 mM Tris pH 7.5, 1 mM EDTA, 1/500 cold protease inhibitor cocktail, [Sigma, St. Louis, MO, USA]). Homogenates were centrifuged $40 \mathrm{~min}$ at $13,000 \mathrm{~g}$ at $4^{\circ} \mathrm{C}$. The supernatant was taken and protein content was determined according to the method of Lowry et al. (1951).

\section{Measurement of HNE-modified proteins by immunoblotting}

Samples containing $75 \mu \mathrm{g}$ of cortex proteins or $50 \mu \mathrm{g}$ plasma proteins were separated by $8 \%$ sodium dodecyl sulfatepolyacrylamide gels electrophoresis (SDS-PAGE), and then transferred to polyvinylidene difluoride membranes.

Membranes were blocked with 5\% skim milk in TBS-T buffer (1 mM Tris, $10 \mathrm{mM} \mathrm{NaCl}$ and $0.5 \%$ Tween 20 ) for $1 \mathrm{~h}$ at room temperature and followed by $1 \mathrm{~h}$ incubation with primary antibodies. Plasmatic proteins were incubated with goat polyclonal anti-HNE antibody (Academy Bio-Medical, Houston, TX, USA) at a dilution of 1:500 and cortex proteins with rabbit polyclonal anti-HNE antibody (Calbiochem, San Diego, CA, USA) at a dilution of 1:1000.

Membranes were washed 3 times in TBS-T buffer for 5 min and then incubated for $1 \mathrm{~h}$ at room temperature, with the corresponding secondary antibody labelled with horseradish peroxidase (HRP) at a dilution of 1:10 000 and washed 3 times in TBS-T buffer for 5 min. Immunoreactivity was detected with chemiluminescence reagent (GE Healthcare, Piscataway, NJ, USA) and probed on X-ray film (UXR, Dorval, QC, Canada). A monoclonal antibody to $\beta$-actin (Sigma, St. Louis, MO, USA) was used at a dilution of 1:200 000 as a control of protein loading.

\section{Detection of carbonyl group on proteins}

Carbonyl groups on proteins were detected by a commercial kit (Oxy Blot ${ }^{\mathrm{TM}}$ Protein Oxidation Detection Kit, Millipore, Billerica, MA, USA). Cortex or plasma protein samples (20 $\mu \mathrm{g}$ ) were mixed with $12 \%$ SDS. Derivation was done by adding 2,4-dinitrophenylhydrazine (DNPH) solution and incubated at room temperature for 15 min and stopped with a neutralization solution. The DNP-derivatized proteins samples were separated by SDS-PAGE followed by western blot using an anti-DNP antibody, at a dilution of 1:300, and with a goat anti-rabbit HRP-conjugated antibody (dilution of 1:300).

\section{RESULTS}

PCA is a widely-used animal model characterized with minimal HE and hyperammonemia (Butterworth et al. 2009). The concentration of ammonia in arterial plasma was significantly higher in PCA vs sham-operated control rats $(142.3 \mu \mathrm{M} \pm 12.0$ vs $83.7 \mu \mathrm{M} \pm 5.4)$. Centrally, in the CSF, ammonia was significantly higher in PCA vs sham-operated controls (135.1 $\mu \mathrm{M} \pm 22.9$ vs $22.0 \mu \mathrm{M} \pm 8.2$ respectively) (Fig. 1).

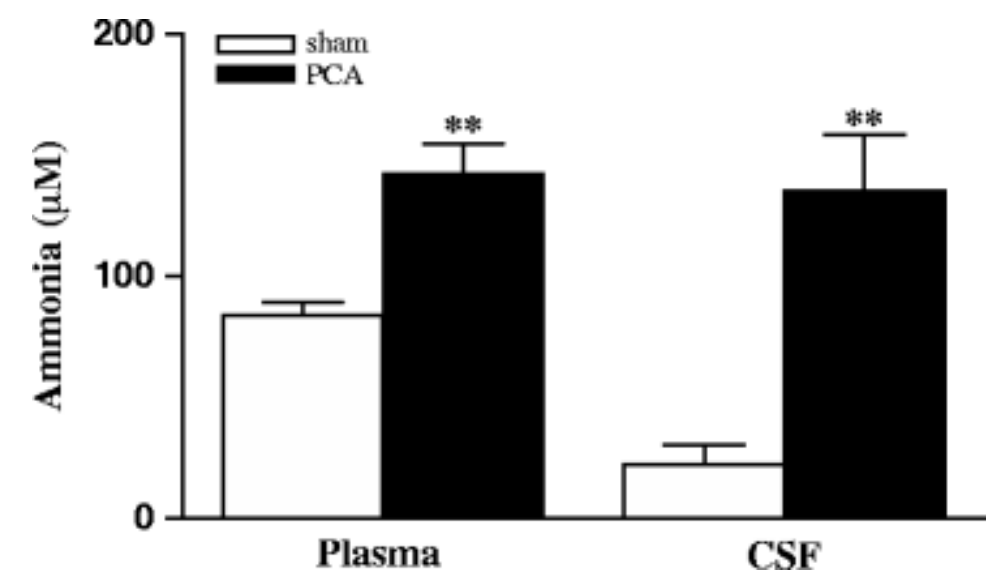

Fig. 1 Ammonia concentrations in plasma and CSF from PCA and sham-operated control rats. Results are expressed as mean \pm S.E.M. ${ }^{* *} \mathrm{p}<0.005$ as compared to sham-operated rats. 
In order to evaluate the status of oxidative stress, HNE-linked proteins and carbonyl levels (oxidative stress markers) on protein side chains were assessed in arterial plasma and frontal cortex tissue by western blot. HNE derivatives did not demonstrate any significant difference in PCA rats compared with sham-operated controls in both arterial plasma (Fig. 2a) and frontal cortex (Fig. 2b). No significant difference was found in carbonyl levels on protein side chains in both arterial plasma (Fig. 3a) and frontal cortex (Fig. 3b), between PCA and sham-operated controls.
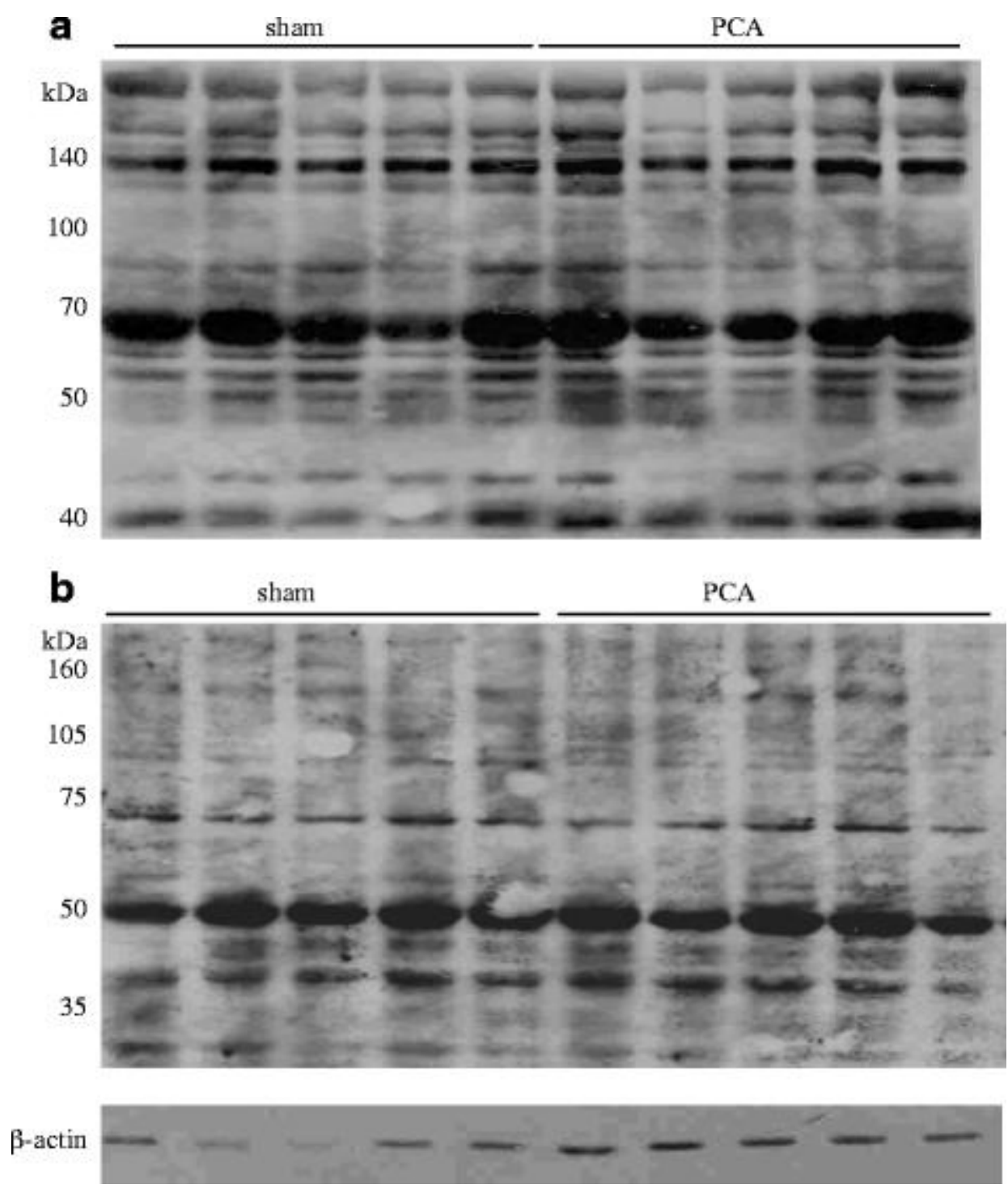

Fig 2. Detection of HNE-linked proteins in arterial plasma (a) and frontal cortex (b) from PCA and sham-operated control rats 


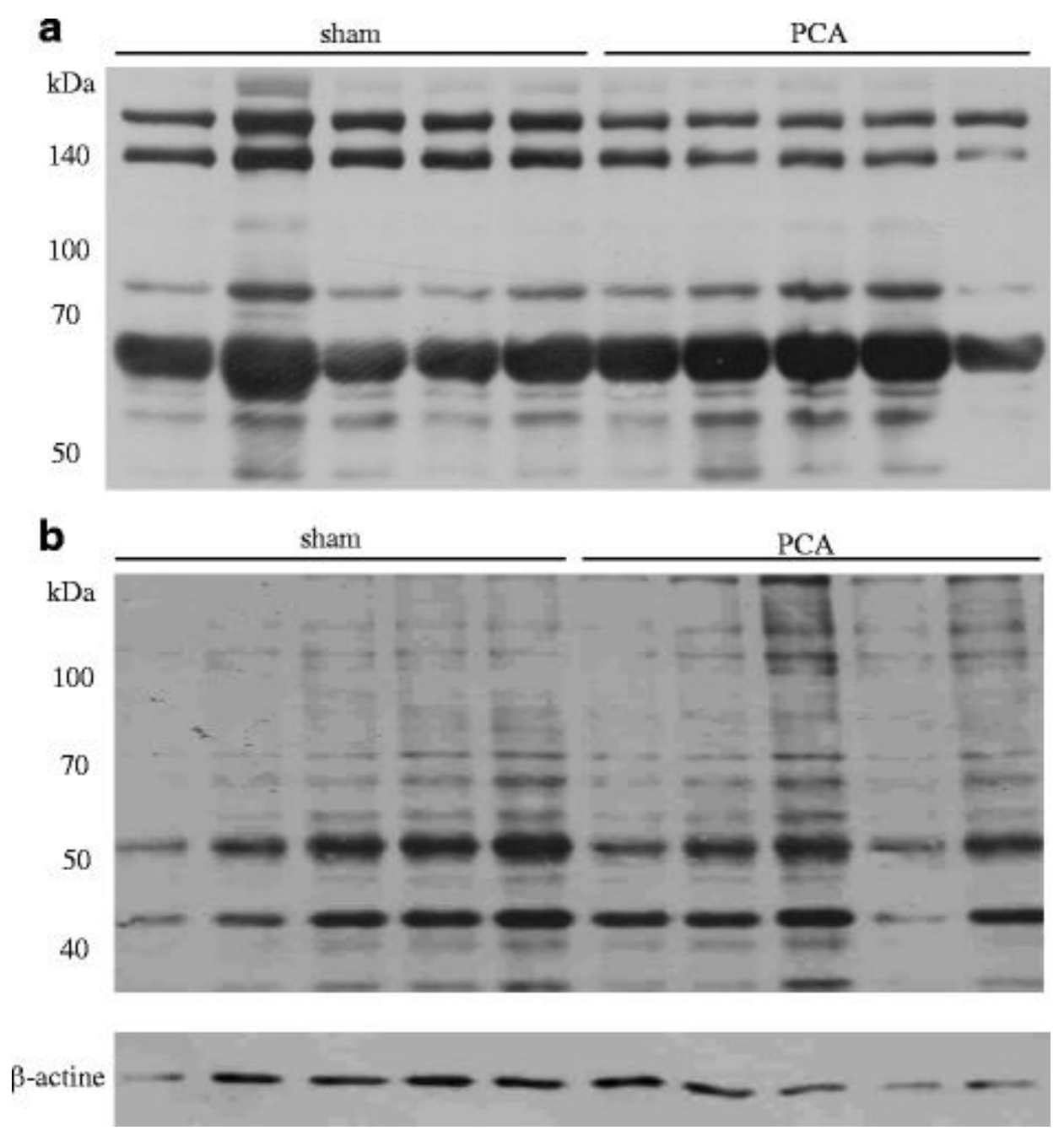

Fig. 3 Detection of carbonyl groups on protein side chains in arterial plasma (a) and frontal cortex (b) from PCA and sham-operated control rats

\section{DISCUSSION}

Hyperammonemia, leading to elevated concentrations of ammonia in the brain, is an important factor in the pathogenesis of HE however its exact mechanisms leading to brain impairment are unclear. PCA shunts gut-derived ammonia from the portal vein to the systemic circulation (inferior vena cava) and results in hyperammonemia and HE, commonly seen in cirrhotic patients following a transjugular intrahepatic portosystemic shunt (Masson et al. 2008). The present study demonstrates that a significant increase in arterial plasma and CSF ammonia levels in PCA rats does not lead to an increase in oxidative stress (HNE and carbonyl derivatives) in both arterial or CSF compared to sham-operated controls.

Recently, oxidative stress has been demonstrated to play a role in the pathogenesis of HE (Norenberg et al. 2004). Cultured astrocytes treated with $5 \mathrm{mM}$ ammonia (for $<24 \mathrm{~h}$ ) lead to an increase in lipid peroxidation and protein carbonyls, two markers of oxidative stress (Norenberg et al. 2007; Widmer et al. 2007). Furthermore, HO-1 expression (mRNA and protein), another marker of oxidative stress, increases following $72 \mathrm{~h}$ of ammonia treatment to cultured astrocytes (Warskulat et al. 2002). Similar results have been found when naïve animals are injected with a high dose of ammonia. Acute ammonia injection to rats leads to an increase in lipid peroxidation, a decrease in antioxidant enzyme activities and in glutathione reserves (Kosenko et al. 2003), along with an increase in HO-1 
Yang, X. et al., 2010. Portacaval anastomosis-induced hyperammonemia does not lead to oxidative stress. Metabolic Brain Disease, 25(1), p.11-15.

protein (Warskulat et al. 2002). The induction of acute ammonia-induced oxidative stress occurs within 11 min following an intraperitoneal injection of $12 \mathrm{mmol} / \mathrm{kg}$ ammonia with an increase in superoxide radical production (Kosenko et al. 2003).

To date, most in vivo studies have associated oxidative stress with HE in models of acute liver failure, which are characterized with hyperammonemia, increased brain ammonia, brain edema, intracranial hypertension, severe HE and death. Oxidative stress has been demonstrated in rats with thioacetamide-induced fulminant hepatic failure (Bruck et al. 1999; Reddy et al. 2004; Sathyasaikumar et al. 2007) with blood and brain ammonia levels of $\sim 1.5 \mathrm{mM}$ and $\sim 710 \mu \mathrm{M}$ respectively (Sathyasaikumar et al. 2007). In our present study, the 4 -week PCA model of chronic hyperammonemia and minimal HE was not accompanied with an induction of oxidative stress. This suggests acute and high ammonia, and not chronic "moderate" hyperammonemia, induces oxidative stress and may be implicated in the pathogenesis of brain edema and/or severe HE.

Ammonia-induced oxidative stress, usually a result of decreased antioxidant capacity (Sathyasaikumar et al. 2007), leads to cellular protein modifications and dysfunction (Norenberg et al. 2004). Hilgier et al. (2003) showed an accumulation of hydroxyl radicals in the microdialysates following ammonia intoxication $(60 \mathrm{mM})$ intrastriatally via a microdialysis probe. This increase was seen at $160 \mathrm{~min}$ following the injection, but returned to baseline levels after $240 \mathrm{~min}$, suggesting this ammonia-induced ROS increase is transient. Numerous studies have demonstrated that treatment with antioxidants against ammonia-induced oxidative stress is very efficacious (Bruck et al. 1999; Guerrini 1994; Widmer et al. 2007) implying oxidative stress is treatable and reversible. However it is uncertain whether the beneficial effect of antioxidants is a direct effect on attenuating oxidative stress or solely by ameliorating liver function and subsequently reducing ammonia.

In present study, oxidative modification of proteins involving HNE derivatives and carbonyl levels in protein side chains in plasma and frontal cortex were unchanged between PCA and sham-operated control rats. This suggests PCA-induced hyperammonemia does not lead to oxidative stress in either blood or brain. One explanation may be due to the subtle increase in blood and brain ammonia. Compared to ammonia-treated induction of oxidative stress in both in vitro and in vivo models, a concentration of $\sim 150 \mu \mathrm{M}$ found in PCA rats is relatively low. Furthermore, oxidative stress may only be implicated in severe HE or death as demonstrated in the acute ammonia- intoxication models compared to the PCA rat, a Type B model of minimal HE (Butterworth et al. 2009). Therefore chronic and moderate hyperammonemia in PCA rats is not suffice to induce oxidative stress. It is important to note, since tyrosine-nitrated proteins have been demonstrated in brains of 4-week PCA rats (Schliess et al. 2002), nitrosative stress' dependence on oxidative stress is undetermined.

In conclusion, a 4-week PCA rat of minimal HE and moderate hyperammonemia didn't express any signs of oxidative stress in either the systemic circulation or the central nervous system. The absence of oxidative stress might be due to a chronic "moderate" elevated ammonia and/or a compensated functional antioxidant system.

\section{REFERENCES}

Blendis L (2006) Hepatic encephalopathy forward to the past. Gastroenterology 130:2239-2240

Bosoi CR, Rose CF (2009) Identifying the direct effects of ammonia on the brain. Metab Brain Dis 24:95-102

Bruck R, Aeed H, Shirin H, Matas Z, Zaidel L, Avni Y, Halpern Z (1999) The hydroxyl radical scavengers dimethylsulfoxide and dimethylthiourea protect rats against thioacetamide-induced fulminant hepatic failure. J Hepatol 31:27-38

Butterworth RF (2003) Hepatic encephalopathy. Alcohol Res Health 27:240-246

Butterworth RF, Norenberg MD, Felipo V, Ferenci P, Albrecht J, Blei AT, HE M of the IC on EM of (2009) Experimental models of hepatic encephalopathy: ISHEN guidelines. Liver Int 29:783-788

Choi JH, Yu BP (1995) Brain synaptosomal aging: free radicals and membrane fluidity. Free Radic Biol Med 18:133-139

Felipo V, Butterworth RF (2002) Neurobiology of ammonia. Prog Neurobiol 67:259-279

The final publication is available at http://dx.doi.org/10.1007/s11011-010-9174-1 
Yang, X. et al., 2010. Portacaval anastomosis-induced hyperammonemia does not lead to oxidative stress. Metabolic Brain Disease, 25(1), p.11-15.

Guerrini VH (1994) Effect of antioxidants on ammonia induced CNS-renal pathobiology in sheep. Free Radic Res 21:35-43

Hazell AS, Butterworth RF (1999) Hepatic encephalopathy: An update of pathophysiologic mechanisms. Proc Soc Exp Biol Med 222:99-112

Hilgier W, Anderzhanova E, Oja SS, Saransaari P, Albrecht J (2003) Taurine reduces ammonia- and N-methyl-D-aspartate-induced accumulation of cyclic GMP and hydroxyl radicals in microdialysates of the rat striatum. Eur J Pharmacol 468:21-25

Kosenko E, Venediktova N, Kaminsky Y, Montoliu C, Felipo V (2003) Sources of oxygen radicals in brain in acute ammonia intoxication in vivo. Brain Res 981:193-200

Lee SH, Fisher B (1961) Portacaval shunt in the rat. Surgery 50:668-672

Lowry OH, Rosebrough NJ, Farr AL, Randall RJ (1951) Protein measurement with the Folin phenol reagent. J Biol Chem 193:265275

Masson S, Mardini HA, Rose JD, Record CO (2008) Hepatic encephalopathy after transjugular intrahepatic portosystemic shunt insertion: a decade of experience. QJM 101:493-501

Norenberg MD, Jayakumar AR, Rama Rao KV (2004) Oxidative stress in the pathogenesis of hepatic encephalopathy. Metab Brain Dis 19:313-329

Norenberg MD, Jayakumar AR, Rama Rao KV, Panickar KS (2007) New concepts in the mechanism of ammonia-induced astrocyte swelling. Metab Brain Dis 22:219-234

Poon HF, Calabrese V, Scapagnini G, Butterfield DA (2004) Free radicals and brain aging. Clin. Geriatr. Med 20:329-359

Reddy PVB, Murthy CRK, Reddanna P (2004) Fulminant hepatic failure induced oxidative stress in nonsynaptic mitochondria of cerebral cortex in rats. Neurosci Lett 368:15-20

Requena JR, Levine RL, Stadtman ER (2003) Recent advances in the analysis of oxidized proteins. Amino Acids 25:221-226

Sathyasaikumar KV, Swapna I, Reddy PVB, Murthy CRK, Gupta AD, Senthilkumaran B, Reddanna P (2007) Fulminant hepatic failure in rats induces oxidative stress differentially in cerebral cortex, cerebellum and pons medulla. Neurochem Res 32:517-524

Schliess F, Görg B, Fischer R, Desjardins P, Bidmon HJ, Herrmann A, Butterworth RF, Zilles K, Häussinger D (2002) Ammonia induces MK-801-sensitive nitration and phosphorylation of protein tyrosine residues in rat astrocytes. FASEB J 16:739-741

Stadtman ER, Levine RL (2003) Free radical-mediated oxidation of free amino acids and amino acid residues in proteins. Amino Acids 25:207-218

Warskulat U, Görg B, Bidmon HJ, Müller HW, Schliess F, Häussinger D (2002) Ammonia-induced heme oxygenase-1 expression in cultured rat astrocytes and rat brain in vivo. Glia 40:324-336

Widmer R, Kaiser B, Engels M, Jung T, Grune T (2007) Hyperammonemia causes protein oxidation and enhanced proteasomal activity in response to mitochondria-mediated oxidative stress in rat primary astrocytes. Arch Biochem Biophys 464:1-11

The final publication is available at http://dx.doi.org/10.1007/s11011-010-9174-1 